\title{
Quantificação de benzeno, tolueno, etilbenzeno e xilenos no ar de ambientes internos
}

\author{
Quantification of benzene, toluene, ethylbenzene \\ and xylenes in the air of indoor environments
}

\section{Paulo Cezar Piceli*, Henrique de Melo Lisboa²}

\section{RESUMO}

A poluição do ar não está restrita a ambientes abertos, podendo existir elevadas concentrações de poluentes do ar derivados do petróleo, como o benzeno, tolueno, etilbenzeno e xilenos (BTEX), em ambientes internos. Os BTEX, mesmo quando presentes em baixas concentrações, na ordem de parte por bilhão (ppb), causam problemas à saúde humana. O objetivo deste trabalho foi aplicar uma técnica de quantificação dos BTEX, no nível de ppb, em apenas oito horas de amostragem em um ambiente interno. Para tal fim, foram usados tubos amostradores passivos associados a análise por dessorção térmica, cromatografia gasosa e espectrometria de massas. O método de calibração desse sistema analítico também foi apresentado. O uso dessa metodologia permitiu quantificar os BTEX em um laboratório de pesquisa de motores de combustão, em concentrações de 4,64, 7,87, 10,47 e 21,36 ppb respectivamente. Esses resultados estão próximos da faixa encontrada em ambientes internos por outros estudos no Brasil. A avaliação dos BTEX no laboratório de combustão, além de levar apenas 16 horas, somando a amostragem e análise das amostras, confirmou a sensibilidade da metodologia usada.

Palavras-chave: poluentes atmosféricos; cromatografia gasosa; espectrometria de massas; injeção direta; amostragem passiva.

\begin{abstract}
Air pollution is not restricted to open air areas. High concentrations of petroleum-based air pollutants may occur, such as benzene, toluene, ethylbenzene and xylenes (BTEX), in indoor environments. The BTEX, even in low concentrations, of the order of parts per billion (ppb), cause human health problems. The objective of this work was to apply a technique of measurement of BTEX, at ppb level, using only eight hours of sampling in an indoor environment. To this end, passive tube samplers were used associated with analysis by thermal desorption, gas chromatography and mass spectrometry. The calibration method applied to this analytical system was also presented. The use of this method allowed the quantification of BTEX in a combustion engines research laboratory, at concentrations of 4.64, 7.87, 10.47 and 21.36 ppb, respectively. These results are close to the range found by other indoor studies in Brazil. The evaluation of BTEX in the combustion laboratory, besides it only takes 16 hours, considering the sampling and the analysis procedures, confirmed the sensitivity of the methodology used.
\end{abstract}

Keywords: air pollutants; gas chromatography; mass spectrometry; direct injection; passive sampling.

\section{INTRODUÇÃO}

A poluição do ar não é restrita a ambientes abertos, ocorrendo significativamente em ambientes internos, ocupacionais ou não (GODISH, 2004). De acordo com a Organização Mundial da Saúde - OMS (WHO, 2014), em cada oito mortes no mundo, uma é causada pela poluição do ar. É afirmado ainda que metade das mortes está relacionada com essa poluição em ambientes internos. Nestes, além da ventilação limitada que diminui a dispersão dos poluentes do ar, atividades como combustão e evaporação podem contribuir para o aumento de suas concentrações (LEE \& CHO, 2002).

Grande parte dos poluentes responsáveis pela redução da qualidade do ar em ambientes internos (QAI) é derivada do petróleo, sendo parte destes denominados compostos orgânicos voláteis (COV). Os COV

podem ser definidos como compostos cujas características físico-químicas tornam possível a sua evaporação sob condições ambientais de temperatura e pressão (USEPA, 2017).

Entre os COV, os poluentes do ar benzeno, tolueno, etilbenzeno e os isômeros de xileno, conhecidos pela sigla BTEX, têm a emissão veicular como sua principal fonte de emissão em centros urbanos (LIN et al., 2011). Wheeler et al. (2013) identificaram que a existência de garagem para carros anexada às residências, fumantes, reformas recentes, uso de removedor de tintas e fragrâncias são fatores que podem contribuir com a presença de BTEX em ambientes internos.

Entre os COV, os isômeros de xileno e o etilbenzeno são as espécies mais reativas. O benzeno é menos reativo e mais estável na atmosfera, 
tendo consequentemente o maior tempo de vida no ambiente (HSIEH \& TSAI, 2003 apud HABEEBULLAH \& HASSANIEN, 2012).

Atualmente, os BTEX têm chamado a atenção da vigilância sanitária devido aos seus efeitos sobre a população afetada. De acordo com a OMS (WHO, 2017) o benzeno é o composto que melhor representa os COV, sendo classificado como cancerígeno aos humanos. O etilbenzeno é rotulado como possivelmente carcinogênico aos humanos, enquanto o tolueno e xilenos não.

Mesmo em baixíssimas proporções no ar, na ordem de parte por bilhão (ppb), os BTEX podem causar irritação e desconforto, devido ao desenvolvimento de sensibilidade das pessoas expostas a eles, com o passar do tempo (BECHER et al., 1996).

No Brasil, não existem limites legais de concentração de BTEX no ar que garantam proteção integral da saúde humana. O que pode se encontrar são limites de tolerância para ambientes ocupacionais, estabelecidos na $15^{\text {a }}$ norma regulamentadora (NR 15) do Ministério do Trabalho e Emprego. Os valores desses limites são estabelecidos visando um equilíbrio entre a proteção dos trabalhadores e a viabilidade econômica de sua aplicação. Foram estabelecidos limites de tolerância de 78 ppm para os compostos tolueno, etilbenzeno e xilenos durante uma jornada de trabalho de 8 horas. Devido às reconhecidas características carcinogênicas aos humanos do benzeno, foi estabelecido, para grande parte das empresas brasileiras, um limite máximo de $1 \mathrm{ppm}$ para o composto. Existem exceções em que, devido a viabilidade técnica, é permitido um limite de tolerância de 2,5 ppm (BRASIL, 1978).

Em levantamento bibliográfico sobre a situação da QAI no Brasil, realizado por Gioda e Aquino Neto (2003), foram encontradas faixas de concentrações entre 0,78 ppb e 7,80 ppb para o benzeno, 55 ppb e $775 \mathrm{ppb}$ para o tolueno e 4,28 ppb e 114 ppb para xilenos. Faixas de concentrações de etilbenzeno em ambientes internos não foram levantadas pelos autores. Esses resultados foram obtidos a partir de outros estudos realizados em diferentes ambientes internos como residências, escritórios, aeroportos, indústrias, gráficas e restaurantes.

Embora a QAI seja um campo de pesquisa emergente no Brasil (COSTA \& COSTA, 2006) e exista uma crescente preocupação com ela, esses fatos não são acompanhados pelo aumento de pesquisas realizadas neste campo (SCHIRMER et al., 2011). Importante salientar que resultados obtidos em pesquisas feitas em outros países não podem simplesmente ser extrapolados para as condições brasileiras, sendo necessárias medições locais. Essa constatação justifica pesquisas nessa área no Brasil.

Para a avaliação da QAI em relação aos BTEX é necessário o desenvolvimento de técnicas de amostragem e análise que produzam resultados confiáveis, rápidos e sensíveis. Segundo a American Society of Heating, Refrigerating and Air-Conditioning Engineers (ASHRAE, 2009), mesmo os compostos considerados como importantes poluentes não são encontrados em níveis acima de 10 ppm. É afirmado ainda que concentrações de COV ficam próximas de 10 pbb. Por essa baixíssima concentração, é justificado o uso de métodos sensíveis de detecção e quantificação destes compostos. Métodos usados em ambientes altamente poluídos, como os ocupacionais em indústrias, geralmente não são apropriados para outros ambientes internos, como escritórios, escolas e residências.

A maioria dos estudos publicados relacionados à análise dos BTEX no ambiente, tanto externo como interno, é focada no desenvolvimento de técnicas de amostragem e de pré-concentração (DEWULF \& LANGENHOVE, 1999).

Amostrar significa segregar uma porção de matéria, neste caso, o ar e seus poluentes, com o objetivo de coletar os compostos para uma análise imediata ou posterior (GODISH, 2004). As técnicas de amostragens podem ser ativas ou passivas. Na ativa, são utilizadas bombas de amostragem para movimentar os poluentes do ar para o amostrador. $\mathrm{Na}$ amostragem passiva, os poluentes são deslocados espontaneamente pela diferença de concentração dos mesmos entre o ar do ambiente analisado e o do interior do amostrador, em um processo denominado difusão (HARRISON \& PERRY, 1986).

A maioria dos amostradores passivos depende da difusão do poluente por meio de uma camada de ar, parada ou estagnada, interna ao amostrador, e da subsequente fixação do poluente em um meio de coleta. Quando um meio coletor adsorvente apropriado ao poluente a ser amostrado é disposto em quantidade adequada dentro do amostrador, a concentração do poluente atmosférico na superfície do adsorvente é considerada nula, formando assim um gradiente de concentração entre a camada de ar no interior do amostrador e o ar ambiente. Cada poluente tem um único coeficiente de difusão no ar. Sendo assim, é transportado para a superfície coletora com uma velocidade dependente de sua concentração no ambiente (HARRISON \& PERRY, 1986). Portanto, quanto maior o gradiente de concentração, maior o fluxo de amostragem do poluente, e vice-versa.

A amostragem passiva é uma técnica de pré-concentração, útil na análise de poluentes atmosféricos orgânicos, especialmente em áreas urbanas. Evoluiu muito nos últimos anos, e quando comparada à amostragem ativa, apresenta como vantagens:

- $\quad$ simplicidade de operação;

- baixo custo;

- desnecessidade de equipamentos acessórios caros e complicados;

- não requerer energia elétrica;

- não necessitar de atenção especial durante a amostragem, sendo capazes atualmente de analisar poluentes em baixa e baixíssima concentrações (KRÓL et al., 2012; ZABIEGALA et al., 2010).

Além disto, ela tem maior aceitação no monitoramento individual, devido ao menor incômodo causado, em comparação ao transporte 
de bombas de amostragem durante 8 horas, em uma jornada de trabalho comum.

A principal desvantagem de seu uso está na necessidade de longos períodos de amostragem que, dependendo do formato do amostrador e do poluente analisado, podem durar semanas, devido à baixa taxa de amostragem desses equipamentos. Por consequência, informações sobre a real exposição de trabalhadores durante uma jornada de trabalho de 8 horas não seria possível. Por exemplo, um longo tempo de amostragem consideraria períodos nos quais as atividades industriais estariam paradas, durante a noite, o que diminuiria as concentrações de BTEX no ambiente, e que por consequência, reduziria a média de concentração a qual o trabalhador estaria realmente exposto. O aperfeiçoamento do formato dos amostradores, aliados a técnicas analíticas sensíveis, como a cromatografia gasosa acoplada à espectrometria de massas (CG-EM), permite a quantificação de COV em concentrações na ordem de ppb.

O objetivo deste estudo foi aplicar uma técnica rápida e sensível de quantificação dos BTEX do ar em ambientes internos, por meio do uso de amostragem passiva e análise por CG-EM. Neste trabalho, um laboratório que pesquisa motores de combustão foi utilizado para a aplicação do procedimento.

\section{METODOLOGIA}

\section{Ambiente interno estudado}

O ar do ambiente interno estudado foi o do Laboratório de Combustão e Engenharia de Sistemas Térmicos (LabCET), localizado no Campus da UFSC, bairro Trindade, na cidade de Florianópolis, Santa Catarina. Nesse laboratório são desenvolvidas pesquisas nas áreas de geração, cogeração e conservação de energia, incluindo geração termelétrica e desenvolvimento de novas tecnologias em processos industriais de combustão.

Na Figura 1 é apresentado o leiaute dos $264 \mathrm{~m}^{2}$ desse laboratório e a localização dos amostradores distribuídos no ambiente.

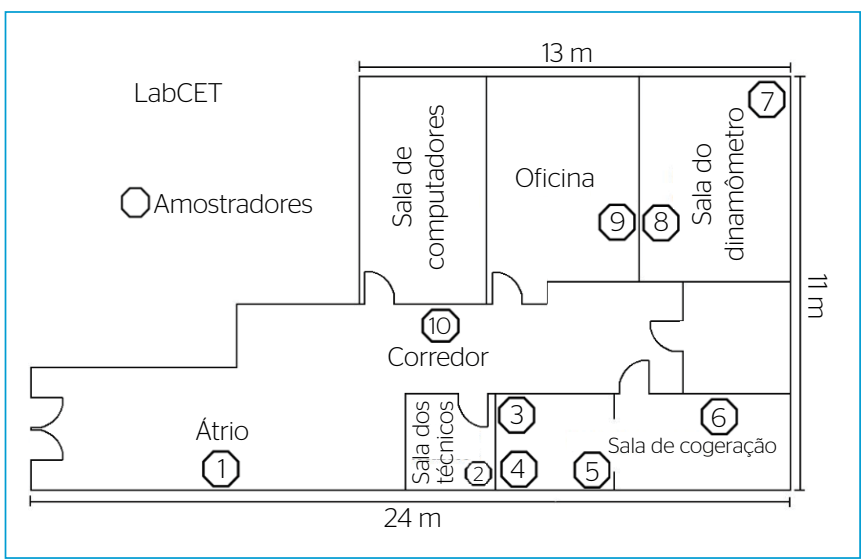

Figura 1 - Leiaute do ambiente interno estudado e localização dos amostradores.
No LabCET, as pesquisas práticas de combustão são realizadas em dois ambientes, chamados de sala de cogeração e do dinamômetro. Na primeira, estão localizados uma turbina movida a gás natural e um motor de combustão interna movido à gasolina. Na sala do dinamômetro estão localizados dois motores para ensaios com diesel ou biodiesel e outro movido a álcool. Por serem ambientes acusticamente isolados, há o acúmulo de BTEX no interior dessas salas, principalmente com a evaporação desses compostos dos motores e tanques de armazenamento de combustíveis. Por consequência, o ar poluído dessas salas é transferido para os ambientes de uso comum do laboratório por difusão, do local mais concentrado para o menos concentrado. Devido ao recesso universitário, as atividades de testes de motores de combustão estavam paradas há mais de 10 dias. Nessa condição, eram esperadas concentrações mínimas de BTEX no ambiente, quando comparadas àquelas provavelmente existentes quando o laboratório está em pleno funcionamento. Dessa forma, foi possível testar a sensibilidade do procedimento analítico proposto para baixas concentrações.

Foram realizadas no LabCET dez amostragens passivas fixas, concomitantes, de 8 horas (480 minutos). Os amostradores foram dispostos nas paredes das salas, presos com fita-crepe, a uma altura de $1,5 \mathrm{~m}$ do solo, ficando assim próximos da zona de respiração dos usuários do laboratório (STRANGER et al., 2008). Os tubos amostradores foram fixados com a superfície de coleta voltada para o chão, evitando a amostragem de material particulado por meio de sedimentação gravitacional.

\section{Preparo dos tubos amostradores}

Em cada tubo amostrador de aço inoxidável, da PerkinElmer (Figura 2), com $8,9 \mathrm{~cm}$ de altura e $0,2 \mathrm{~cm}^{2}$ de área transversal (A), foram acomodados $200 \mathrm{mg}$ de carvão ativado (granulometria entre 20 a 40 mesh e área superficial de $100 \mathrm{~m}^{2} \cdot \mathrm{g}^{-1}$ ), formando um leito adsorvente de $6 \mathrm{~cm}$ de altura no interior do tubo e percurso de difusão $(\mathrm{Z})$ de $1,5 \mathrm{~cm}$.

Após a montagem, cada tubo amostrador foi condicionado em condições extremas, com a passagem de gás hélio $\left(100 \mathrm{ml} \cdot \mathrm{min}^{-1}\right)$ a

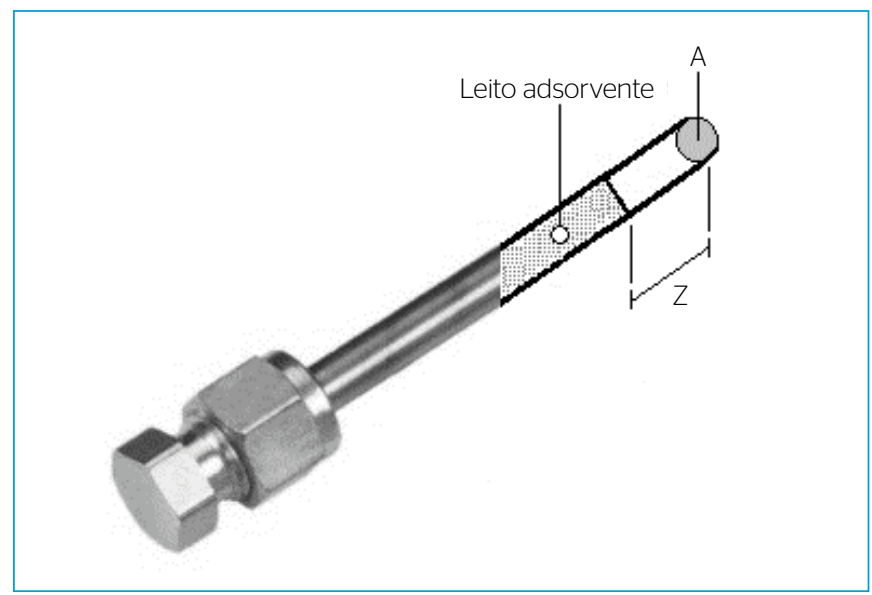

Figura 2 - Tubo amostrador Perkin Elmer. 
uma temperatura de $350^{\circ} \mathrm{C}$ (máximo suportado pelo adsorvente) durante 2 horas, como o preconizado pela United States Environmental Protection Agency (USEPA, 1999). Esse procedimento foi realizado em um dessorvedor térmico automatizado, modelo Thermodesorber, da marca PerkinElmer.

\section{Armazenamento das amostras}

$\mathrm{O}$ armazenamento das amostras foi feito de acordo com o método proposto por Peng e Batterman (2000). Após a amostragem, cada tubo amostrador foi tampado, envolvido com uma folha de alumínio, sendo mantido em recipiente de vidro limpo, previamente lavado com água deionizada e seco em estufa a aproximadamente $100^{\circ} \mathrm{C}$, e selado com filme plástico.

Dois amostradores foram utilizados como brancos de campo. Eles foram embalados com a folha de alumínio no laboratório, após o processo de limpeza por dessorção, sendo em seguida colocados no recipiente de armazenamento. No local de amostragem, os amostradores foram desembalados e imediatamente embalados com papel-alumínio novamente, sendo então depositados no recipiente de armazenamento (ANDRIETTA et al., 2010). Com exceção da etapa de amostragem, esse procedimento expôs os brancos de campo às mesmas condições aplicadas aos amostradores, permitindo a detecção de possíveis contaminações durante o armazenamento das amostras.

\section{Sistema analítico}

Utilizou-se, neste trabalho, um sistema analítico composto por um dessorvedor térmico, acoplado a um cromatógrafo a gás, com detector de espectros de massas.

A dessorção térmica dos poluentes retidos nos amostradores foi realizada em um dessorvedor térmico automatizado (Thermodesorber da PerkinElmer). Cada dessorção foi efetuada automaticamente, em dois estágios pré-programados. No primeiro estágio, os analitos foram extraídos dos tubos amostradores, com um fluxo de 100 mL.min ${ }^{-1}$ de gás hélio, aquecido a uma temperatura de $325^{\circ} \mathrm{C}$, durante 10 minutos e transferidos para uma armadilha fria, mantida a $-30^{\circ} \mathrm{C}$. Na segunda dessorção, a armadilha fria foi aquecida a uma taxa de $40^{\circ} \mathrm{C} . \mathrm{s}^{-1}$ até atingir $325^{\circ} \mathrm{C}$, transferindo $80 \%$ das amostras (divisão de fluxo de $20 \mathrm{~mL} \cdot \mathrm{min}^{-1}$ ) para a coluna cromatográfica. A baixa taxa de divisão (1:5) fez com que a maior parte das amostras chegasse ao detector não comprometendo o sistema analítico e aumentando a sensibilidade do mesmo, pois foram analisadas pequenas massas de poluentes adsorvidos nos amostradores.

Após a dessorção dos analitos, estes foram transferidos diretamente para a separação em um cromatógrafo a gás (Autosystem XL da PerkinElmer), utilizando uma vazão de hélio a 1,30 $\mathrm{mL} \cdot \mathrm{min}^{-1} \mathrm{em}$ uma coluna Cromacon Ciola 5 (30 $\mathrm{m}$ de comprimento, 0,25 $\mathrm{mm}$ de diâmetro interno e de $0,5 \mu \mathrm{m}$ de espessura de fase estacionária). Para o aquecimento da coluna, foi elaborada uma rampa de calefação, iniciada em uma temperatura de $55^{\circ} \mathrm{C}$, por 5 minutos. Na sequência, a coluna foi aquecida até $160^{\circ} \mathrm{C}$, a uma taxa de $45^{\circ} \mathrm{C} \cdot \mathrm{min}^{-1}$, para então ser esquentada lentamente até $320^{\circ} \mathrm{C}$, a uma taxa de $6^{\circ} \mathrm{C} \cdot \mathrm{min}^{-1}$. O tempo de corrida foi de 34 minutos, acrescido de 10 minutos para o resfriamento do forno, à temperatura inicial de $55^{\circ} \mathrm{C}$.

A detecção dos poluentes, após a eluição da coluna cromatográfica, foi realizada em um espectrômetro de massas do tipo quadrupolo (TurboMass da PerkinElmer). A ionização elétrica foi realizada em uma temperatura de $150^{\circ} \mathrm{C}$, no modo de varredura total dos espectros (full scan), com relação de massa sobre carga (m/z) variando entre 45 200, a cada 0,4 segundos, com intervalo de $0,05 \mathrm{seg}^{-1}$ entre cada espectro.

\section{Calibração do sistema analítico}

Para a calibração do sistema analítico e a posterior quantificação dos resultados, foi realizado o método de injeção direta descrito pela Health Safety Executive (1997). Foram feitas injeções nos tubos adsorventes, previamente purgados no dessorvedor térmico, de alíquotas de 1, 3 e $5 \mu \mathrm{L}$ de soluções-padrão de BTEX com concentrações de 100, 10 e $1 \mu \mathrm{g} \cdot \mathrm{mL}^{-1}$ cada, totalizando 9 pontos nas curvas de calibração. As soluções de 10 e $1 \mu \mathrm{g} \cdot \mathrm{mL}^{-1}$ foram preparadas a partir da solução de BTEX de $100 \mu \mathrm{g} \cdot \mathrm{mL}^{-1}$.

Após a realização de cada injeção, foi realizada uma purga com ar comprimido, filtrado por filtro de carvão ativado, com vazão de $100 \mathrm{~mL} \cdot \mathrm{min}^{-1}$, durante 2 minutos. Essa técnica permitiu a volatilização do solvente e analitos, forçando a passagem destes sobre o leito adsorvente, em fase gasosa. Esse procedimento ainda permitiu a volatilização e descarte do excesso de metanol, evitando a queima do filamento se uma quantidade significativa desse solvente chegasse ao detector de massas (SOUSA, 2002).

A injeção de nove alíquotas de soluções-padrão em tubos adsorventes resultou em diferentes respostas geradas pelo sistema analítico para as massas (em nanogramas) de BTEX, formando posteriormente as curvas de calibração para quantificação desses poluentes no ar. Cada ponto da curva foi feito em duplicata (SOARES, 2001). Critérios de qualidade sobre esses pontos foram aplicados com o objetivo de ajustar as variáveis estatísticas das curvas de calibração, de acordo com o determinado por Pimentel e Barros Neto (1996). Os cálculos foram desenvolvidos no software Microsoft Excel 11.0.

Os limites de detecção e quantificação do método de injeção direta foram calculados por método baseado em parâmetros da curva analítica, em ppb, como o indicado pela norma DIN 2449 da Verein Deutscher Ingenieure (VDI, 1995).

No caso de obtenção de respostas inferiores aos limites de quantificação determinados, a concentração do composto analisado foi considerada igual à metade do valor do limite de detecção estipulado para o poluente (BRASIL, 1995). 


\section{Taxa de amostragem passiva}

Foram determinadas taxas de amostragem passivas teóricas de BTEX, baseadas na Primeira Lei de Fick, conforme o relatado por Andrietta et al. (2010). As taxas de amostragem $\left(\mathrm{U}_{\mathrm{p}}\right)$ foram calculadas em nanogramas por parte por milhão por minuto $\left(\mathrm{ng} \cdot \mathrm{ppm}^{-1} \cdot \mathrm{min}^{-1}\right)$, utilizando a Equação 1:

$U_{p}=\frac{60 \times D_{a r} \times A \times P M}{24,45 \times Z}$

Em que:

$\mathrm{D}_{\mathrm{ar}}=\mathrm{o}$ coeficiente de difusão do composto no $\operatorname{ar}\left(\mathrm{cm}^{2} \cdot \mathrm{s}^{-1}\right)$;

$\mathrm{A}=$ área da seção transversal do percurso de difusão $\left(\mathrm{cm}^{2}\right)$;

$\mathrm{Z}=$ comprimento do percurso de difusão do tubo $(\mathrm{cm})$;

$\mathrm{PM}=$ peso molecular do composto $(\mathrm{g})$.

Para a determinação de $\mathrm{D}_{\text {ar }}$, foram utilizados os dados obtidos experimentalmente por Lugg (1968), que estudou 147 compostos orgânicos a uma pressão de $1 \mathrm{~atm}(760 \mathrm{mmHg})$ e $25^{\circ} \mathrm{C}$. Os coeficientes de difusão para o benzeno, tolueno, etilbenzeno e os isômeros de xilenos utilizados são apresentados no Quadro 1.

$\mathrm{OD}_{\mathrm{ar}}$ dos xilenos foi definido como a média dos valores apresentados por Lugg (1968) para os três isômeros de xileno (orto, para e meta). A utilização dos valores experimentais, no cálculo das taxas de amostragem dos poluentes do ar BTEX, é mais precisa do que coeficientes de difusão, que poderiam ser calculados teoricamente conforme proposto por Fuller (1966 apud SCHWARZENBACH et al., 2003). É recomendável a utilização de valores teóricos dos coeficientes de difusão $\left(\mathrm{D}_{\mathrm{ar}}\right)$ apenas para poluentes cujos coeficientes de difusão não são encontrados na literatura.

\section{Cálculo das concentrações de BTEX no ar}

Após as análises das amostras com a quantificação da massa adsorvida do analito no adsorvente e as taxas de amostragem (Up) definidas, foi então utilizada a Equação 2 para o cálculo das concentrações do BTEX em parte por milhão (ppm):

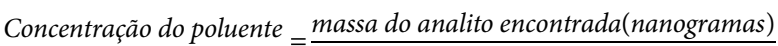
no $\operatorname{ar}(p p m) \quad=\frac{\text { Up } \times \text { Tempo de amostragem(minutos) }}{}$

Quadro 1 - Coeficientes de difusão em $\mathrm{cm}^{2} \cdot \mathrm{s}^{-1}$.

\begin{tabular}{|l|c|}
\hline Compostos & $\mathrm{D}_{\text {ar }}$ médio do experimento $\left(\mathrm{cm}^{2} \cdot \mathrm{seg}^{-1}\right)$ \\
\hline Benzeno & 0,0932 \\
\hline Tolueno & 0,0849 \\
\hline Etilbenzeno & 0,0755 \\
\hline Xilenos & 0,0695 \\
\hline
\end{tabular}

Fonte: adaptado de Lugg (1968).

\section{RESULTADOS E DISCUSSÃO}

Os resultados obtidos abrangem o perfil cromatográfico, calibração do sistema analítico, por meio da análise de regressão, a um índice de confiança de $95 \%$.

\section{Perfil cromatográfico}

Os tempos de eluição dos BTEX da coluna cromatográfica, com a aplicação da rampa de aquecimento do forno, corresponderam, em média, à metade dos valores citados por Sousa (2002). Esse fato pode ser explicado pelo uso de uma rampa menos tênue, com taxa de aquecimento maior e uma temperatura inicial de $55^{\circ} \mathrm{C}$. Os tempos de eluição dos compostos estudados não apresentaram variações consideráveis entre as análises: benzeno, entre 2,68 e 2,69 minutos; tolueno, 4,39 e 4,46 minutos; etilbenzeno, 6,34 e 6,42 minutos; $m$ e $p$-xilenos, , 46 e 6,51 minutos; e $o$-xileno, 6,74 e 6,76 minutos.

A análise dos brancos das amostras de campo não apresentou níveis de contaminação acima dos limites de detecção estabelecidos, dispensando o débito dos valores de contaminação de BTEX dos resultados das amostras.

\section{Calibração do sistema analítico}

Para cada poluente analisado, foi construída uma curva de calibração, formada por 9 pontos, que foram determinados por meio da injeção de diferentes concentrações, ou massas dos analitos, nos tubos amostradores, em duplicata. Conhecida a massa injetada nos amostradores (ng), a taxa de amostragem passiva do poluente $\left(\mathrm{U}_{\mathrm{p}}\right)$ e o tempo de amostragem usado (min), e aplicando esses valores na Equação 2, foi possível colocar como variável explanatória, ou independente das curvas, a concentração do poluente em ppb. As respostas pelo sistema analítico, após análise dos tubos amostradores, foram consideradas variáveis independentes das curvas.

No Quadro 2 são apresentadas as variáveis estatísticas ajustadas, utilizadas na construção da curva de calibração, por meio do método de injeção direta.

Observando os resultados do Quadro 2, nota-se uma correlação linear positiva entre a concentração equivalente à massa adsorvida nos amostradores e a resposta do detector de massas, demonstrando que a regressão é significativa. De acordo com a Verein Deutscher Ingenieure

Quadro 2 - Curva de calibração dos BTEX por injeção direta.

\begin{tabular}{|l|c|c|c|}
\hline Composto & $\begin{array}{c}\text { Coeficiente de } \\
\text { determinação }\left(r^{2}\right)\end{array}$ & $\begin{array}{c}\text { Coeficiente de } \\
\text { correlação }(r)\end{array}$ & Equação da reta \\
\hline Benzeno & 0,9767 & 0,9883 & $y=30,979+13,417^{*} x$ \\
\hline Tolueno & 0,9767 & 0,9883 & $y=72,546+27,185^{*} x$ \\
\hline Etilbenzeno & 0,9820 & 0,9910 & $y=147,676+27,331^{*} x$ \\
\hline Xilenos & 0,9814 & 0,9906 & $y=22,974+28,024^{*} x$ \\
\hline
\end{tabular}


(1995), isso significa que o processo de preparo e injeção das soluções foi realizado com precisão e repetibilidade. Interessante observar que as curvas não foram forçadas a passar pela origem do plano cartesiano $(0,0)$. Segundo Pimentel e Barros Neto (1996), essa seria uma ação desnecessária, pois seria suposto que o ponto origem tem uma variância igual a zero, o que dificilmente ocorre em técnicas sensíveis de detecção como a espectrometria de massas. Observou-se que mesmo para os níveis de brancos, traços de alguns compostos foram encontrados, incluindo os BTEX. Isso ocorreu, provavelmente, devido à degradação térmica do adsorvente carvão ativado durante a dessorção.

Os limites de detecção calculados foram de 0,64 ppb, 0,15 ppb, 1,02 ppb e 0,07 ppb para os poluentes do ar benzeno, tolueno, etilbenzeno e xilenos, respectivamente. Embora tenham sido obtidos limites baixíssimos de detecção, apenas dos isômeros de xileno ficou abaixo de 0,5 ppb recomendado pelo método TO-17 da USEPA (1999). O uso do método monitoramento seletivo de íons em vez da varredura total dos espectros poderia auxiliar na redução dos limites de detecção do espectrômetro de massas para os BTEX. Os limites de quantificação estipulados foram de 1,28 ppb para o benzeno, de 0,31 ppb para o tolueno, de 2,04 para o etilbenzeno e 0,15 para os isômeros de xileno.

\section{Análise do ar do ambiente interno}

Com o uso da Equação 1 foram elaborados os cálculos das taxas de amostragens ideais $\left(U_{p}\right)$ dos tubos amostradores em relação aos poluentes estudados (Quadro 3).

As taxas de amostragem apontadas no Quadro 3 são baseadas em coeficientes de difusão experimentais $\left(\mathrm{D}_{\mathrm{ar}}\right)$ obtidos a uma temperatura de $25^{\circ} \mathrm{C}$ e 1 atm, condições estas similares aos do ambiente estudado. Para condições diferentes de pressão e temperatura, é recomendável fazer as correções necessárias nos valores de $\mathrm{D}_{\mathrm{ar}}$.
A aplicação do método de amostragem passiva, somada à calibração do sistema analítico por injeção direta, permitiu a quantificação das concentrações de BTEX no ar do laboratório estudado. Os resultados obtidos são mostrados na Tabela 1 e refletem a exposição de funcionários e estudantes do local aos BTEX, em ppb, durante uma jornada de 8 horas.

As concentrações de BTEX no ar, obtidas neste estudo, são provavelmente resultantes de emissão fugitiva por evaporação dos combustíveis, nos motores e reservatórios de combustíveis, e pela liberação destes compostos eventualmente adsorvidos das superfícies do ambiente analisado, como, por exemplo, as paredes do laboratório.

Comparando as médias de concentrações de benzeno, tolueno e xilenos, no ar do laboratório, com as obtidas no estudo brasileiro de Gioda e Aquino Neto (2003), nota-se que os valores médios de benzeno e xilenos estão dentro da faixa levantada em pesquisas feitas em ambientes internos no país. Entretanto, a concentração de tolueno está abaixo do mínimo de 55 ppb, levantado por estes mesmos autores. Porém, concentrações mais elevadas de BTEX são esperadas durante o pleno funcionamento do laboratório, com a realização dos testes com motores de combustão.

É importante observar que para o poluente benzeno, foram detectadas em 5 amostras, concentrações abaixo do limite de quantificação

Quadro 3 - Taxas de amostragem ideais calculadas para tubos difusivos da PerkinElmer.

\begin{tabular}{|l|c|}
\hline Compostos & $\mathrm{U}_{\mathrm{p}}\left(\mathrm{ng} \cdot \mathrm{ppm}^{-1} \cdot \mathrm{min}^{-1}\right)$ \\
\hline Benzeno & 2,4436 \\
\hline Tolueno & 3,0723 \\
\hline Etilbenzeno & 2,6358 \\
\hline Xilenos & 2,4263 \\
\hline
\end{tabular}

Tabela 1 - Resultados em ppb obtidos por meio da quantificação de BTEX no ar do LabCET.

\begin{tabular}{|c|c|c|c|c|c|}
\hline Amostra & Local & Benzeno & Tolueno & Etilbenzeno & Xilenos \\
\hline (1) & Átrio & 0,32 & 1,64 & 21,45 & 26,48 \\
\hline (2) & Sala dos técnicos & 0,32 & 3,33 & 22,48 & 25,16 \\
\hline (3) & Sala Cogeração Motor Combustão Interna & 22,51 & 32,02 & 13,88 & 44,00 \\
\hline (4) & Sala Cogeração Motor Combustão Interna & 11,56 & 11,64 & 4,59 & 21,55 \\
\hline (5) & Sala Cogeração Motor Combustão Interna & 6,86 & 8,85 & 6,86 & 22,55 \\
\hline (6) & Sala Cogeração Turbina & 0,32 & 3,55 & 0,51 & 13,49 \\
\hline (7) & Sala do dinamômetro & 0,32 & 3,22 & 5,87 & 11,85 \\
\hline (8) & Sala do dinamômetro & 1,12 & 3,84 & 7,40 & 14,34 \\
\hline (9) & Oficina & 0,32 & 3,33 & 8,98 & 14,52 \\
\hline \multirow[t]{4}{*}{ (10) } & Corredor & 2,76 & 3,00 & 12,75 & 19,63 \\
\hline & Limite de detecção & 0,64 & 0,15 & 1,02 & 0,07 \\
\hline & Limite de quantificação & 1,28 & 0,31 & 2,04 & 0,15 \\
\hline & Média das concentrações & 4,64 & 7,87 & 10,47 & 21,36 \\
\hline
\end{tabular}


de 1,28 ppb. Embora esperado resultados baixíssimos para benzeno, visto que o uso deste composto é permitido apenas em pouquíssimas concentrações no Brasil, não se pode considerar que ele não exista no ar dos pontos de amostragem estudados, justificando o uso do valor de 0,32 ppb, conforme o sugerido em Brasil (1995). Esse procedimento evitou a subavaliação da concentração média do benzeno.

Mesmo nas baixas concentrações amostradas, são esperados irritação e desconforto das pessoas que ficam presentes neste local, resultante do aumento da sensibilidade pessoal após exposição, conforme o relatado por Becher et al. (1996). Até durante o recesso das atividades do laboratório, o acionamento do sistema de ventilação e exaustão dos poluentes neste ambiente, a extração de combustíveis remanescentes do interior dos motores e o estancamento de emissões por evaporação dos tanques de armazenamento de combustível, são medidas que possibilitariam a melhoria da qualidade do ar no local.

As vantagens observadas da aplicação do método aqui utilizado foram a rapidez da etapa de amostragem e análise e o alcance de quantificação de BTEX em ambientes internos na ordem de ppb. Não considerando o tempo de calibração do sistema analítico, a amostragem e as análises das amostras foram realizadas em aproximadamente 16 horas, possibilitando uma rápida avaliação do ambiente e a aplicação de possíveis medidas de diminuição das concentrações dos poluentes.

\section{CONCLUSÃO}

A quantificação dos poluentes BTEX no ar de um ambiente interno permitiu avaliar a exposição de estudantes e funcionários de um laboratório de motores de combustão durante o período de oito horas em nível de ppb.

Os resultados médios de concentração dos poluentes do ar benzeno, tolueno, etilbenzeno e xilenos, no laboratório, foram de 4,64 ppb,
7,78 ppb, 10,47 ppb e 21,36 ppb, respectivamente. Estes níveis de BTEX estão muito abaixo dos limites legais da NR 15, podendo o laboratório ser considerado seguro em relação ao risco de câncer e outras doenças associadas aos BTEX no ar. Entretanto, os teores de BTEX podem ser provavelmente maiores quando o laboratório investigado retomar o ritmo normal de atividades e pesquisas com combustão. Porém, essas informações podem servir de base para justificar ações de remediação necessárias em prol da melhoria da qualidade do ar no local, evitando desconforto e danos à saúde pela presença dos poluentes do ar estudados. É enfatizado que valores muito acima destas médias são esperados quando houver atividades de pesquisa em testes de combustão no laboratório.

Para uma análise efetiva da exposição ao BTEX, é aconselhada a utilização de amostradores fixos no ambiente e a fixação dos amostradores diretamente em funcionários e estudantes, próximos de suas zonas de respiração, durante suas jornadas de trabalho. São sugeridas novas avaliações de quantificação desses poluentes do ar, com o ambiente laboral funcionando normalmente, em diferentes dias da semana, incluindo análises em diferentes estações do ano, quando se espera o aumento das concentrações obtidas e a consequente piora da qualidade do ar interno.

Ainda é aconselhada a redução dos limites de detecção e quantificação do benzeno para o método aplicado, usando a injeção de menores concentrações de benzeno nos tubos amostradores, sensibilizando a análise da concentração deste poluente no ar.

A aplicação das metodologias expostas de amostragem passiva, uso do dessorvedor térmico automatizado, do cromatógrafo a gás e do espectrômetro de massas, bem como a calibração do sistema analítico por injeção direta, podem ser testadas em estudos futuros, quantificando outros compostos orgânicos voláteis, emitidos no ar de outros ambientes internos.

\section{REFERÊNCIAS}

AMERICAN SOCIETY OF HEATING, REFRIGERATING AND AIRCONDITIONING ENGINEERS (ASHRAE). (2009) Indoor air quality guide: best practices for design, construction and commissioning. Atlanta, GA: ASHRAE. 640 p.

ANDRIETTA, L.C.G.; TOMAZ, E.; TRESMONDI, A.C.C.L.; CREMASCO, M.A. (2010) Experimental determination of benzene uptake rate in Tenax TA diffusive samplers. Química Nova, v. 33, n. 5, p. 1034-1038. http://dx.doi.org/10.1590/S0100-40422010000500005

BECHER, R.; HONGSLO, J.K.; JANTUNEN, M.J.; DYBING, E. (1996) Environmental chemicals relevant for respiratory hypersensitivity: the indoor environment. Toxicology Letters, n. 86, p. 155-162. https:// doi.org/10.1016/0378-4274(96)03685-5
BRASIL. Ministério do Trabalho e do Emprego (MTE). (1978) Norma Regulamentadora 15 - Atividades e Operações Insalubres, 6 julho de 1978. Disponível em: <http://portal.mte. gov.br/images/Documentos/SST/NR/NR15/NR15-ANEX015.pdf> Acesso em: 3 mar. 2017.

Secretaria de Segurança e Saúde no Trabalho. (1995) Instrução Normativa no 1, de 20 de dezembro de 1995. Avaliação das concentrações de benzeno em ambientes de trabalho. Brasília: Secretaria de Segurança e Saúde no Trabalho.

COSTA, M.F.B.; COSTA, M.A.F. (2006) A qualidade do ar de interiores e a saúde humana. Revista de Gestão Integrada em Saúde do Trabalho e Meio Ambiente, São Paulo, v. 1, n. 2, p. 1-10. 
DEWULF, J.; LANGENHOVE, H.V. (1999) Anthropogenic volatile organic compounds in ambient air and natural waters: a review on recent developments of analytical methodology, performance and interpretation of field measurements. Journal of ChromatographyA, n. 843, p. 163-177.

GIODA, A.; AQUINO NETO, F.R. (2003) Poluição química relacionada ao ar de interiores no Brasil. Química Nova, v. 26, p. 359-365. http:// dx.doi.org/10.1590/S0100-40422003000300013

GODISH, T. (2004) Air quality. 4. ed. Boca Raton, FL: CRC Press. 480 p.

HABEEBULLAH, T.; HASSANIEN, M.A. (2012) Determination of BTEX pollutants in ambient air of the holy city of Makkah. The Meteorology, Environment and Arid Land Agriculture Journal of King Abdulaziz University, v. 23, n. 2, p. 91-101. https://doi.org/ 10.4197/Met. 23-2-6

HARRISON, R.M.; PERRY, R. (1986) Handbook of air pollution analysis. 2. ed. Londres, Reino Unido: Chapman and Hall. 506 p.

HEALTH AND SAFETY EXECUTIVE. (1997) Methods for the determination of hazardous substances 33/2 - sorbent tube standards. Reino Unido: Health and Safety Executive Publisher.

KRÓL, S.; ZABIEGALA, B.; NAMIÉSNKI, J. (2012) Measurement of benzene concentration in urban air using passive sampling. Analytical and Bioanalytical Chemistry, v. 403, p. 1067-1082. https:// doi.org/10.1007/s00216-011-5578-y

LEE, B.K.; CHO, J. (2002) Risk of analysis of volatile organic compounds through daily life cycle in the industrial city in Korea. Water, Air, and Soil Pollution: Focus, v. 2, p. 155-171. https://link. springer.com/article/10.1023/A:1021375015671

LIN, C.; LIN, C.; HSIEH, L.; CHEN, C.; WANG, P.(2O11) Vertical and Diurnal Characterization of Volatile Organic Compounds in Ambient Air in Urban Areas. Journal of the Air \& Waste Management Association, v. 61, p. 714-720. https://doi.org/10.3155/1047-3289.61.7.714

LUGG, G.A. (1968) Diffusion coefficients of some organic and other vapors in air. Analytical Chemistry, v. 40, n. 7. https://doi.org/ 10.1021/ ac60263aO06

PENG, C.Y.; BATTERMAN, S. (2000) Performance evaluation of a sorbent tube sampling method using short path thermal desorption for volatile organic compounds. Journal of Environmental Monitoring, n. 2, p. 313-324. https://doi.org/10.1039/ B003385P

PIMENTEL, M.F.; BARROS NETO, B. (1996) Calibração: uma revisão para químicos analíticos. Revisão. Química Nova, v. 19, n. 3, p. 268-277.
SCHIRMER, W.N.; PIAN, L.B.; SZYMANSKI, M.S.E.; GAUER, M.A. (2O11) A poluição do ar em ambientes internos e a síndrome dos edifícios doentes. Ciência \& Saúde Coletiva, v. 16, n. 8, p. 3583-3590. http:// dx.doi.org/10.1590/S1413-81232011000900026

SCHWARZENBACH, R.P.; GSCHWEND, P.M.; IMBODEN, D.M. (2003) Environmental Organic Chemistry. 2. ed. Nova York, NY: WileyInterscience. $1313 \mathrm{p}$.

SOARES, L.M.V. (2011) Como obter resultados confiáveis em cromatografia. Revista Instituto Adolfo Lutz, v. 60, n. 1, p. 79-84.

SOUSA, K.R.P. (2002) Estudo sobre compostos orgânicos voláteis presentes no ar do município de Paulínia. Dissertação (Mestrado) Universidade Estadual de Campinas, Campinas.

STRANGER, M.; POTGIETER-VERMAAK, S.S.; VAN GRIEKEN, R. (2008) Characterization of indoor air quality in primary schools in Antwerp, Belgium. Indoor Air, n. 18, p. 454-463. https://doi.org/10.1111/ j.1600-0668.2008.00545.x

U. S. ENVIRONMENTAL PROTECTION AGENCY (USEPA). (1999) Compendium of Methods for the Determination of Toxic Organic Compounds in Ambient Air: Method TO-17. Estados Unidos. U. S. Environmental Protection Agency, Research Triangle Park, USEPA, 1999.

. Volatile Organic Compounds (VOCs). Disponível em: <https:// www.epa.gov/indoor-air-quality-iaq/technical-overview-volatileorganic-compounds>. Acesso em: 3 maio 2017.

THE ASSOCIATION OF GERMAN ENGINEERS (VDI). (1995) DIN 2449: Determination of Performance Characteristics for Measurement of Gaseous Pollutants (Immission). Part 1.

WHEELER, A.J.; WONG, S.L.; KHOURY, C.; ZHU, J. (2O13) Predictors of indoor BTEX concentrations in Canadian residences. Health Reports, v. 24, n. 5.

WORLD HEALTH ORGANIZATION (WHO). (2014) Ambient (outdoor) air quality and health. Fact Sheet n. 313. Disponível em: <http://www.who.int/mediacentre/factsheets/fs313/en/>. Acesso em: 3 mar. 2017.

International Agency for Research on Cancer (IARC). Monographs on the Evaluation of Carcinogenic Risks to Humans. Disponível em: <http://monographs.iarc.fr/ENG/Classification/ index.php>. Acesso em: 3 mar. 2017.

ZABIEGALA, B.; KOT-WASIK, A.; URBANOWICZ, M.; NAMIÉSNIK, J. (2010) Passive sampling as a tool for obtaining reliable analytical information in environmental quality monitoring. Analytical and Bioanalytical Chemistry, v. 396, p. 273-296. https://doi.org/10.1007/ s00216-009-3244-4 